\title{
The Dynamic Competition Between Stress Generation and Relaxation Mechanisms During Coalescence of Volmer-Weber Thin Films.
}

J. A. Floro, S. J. Hearne, J. A. Hunter, and P. Kotula

Sandia National Laboratories, Albuquerque, NM 87185-1415

E. Chason

Division of Engineering, Brown University, Providence, RI 02912

S. C. Seel and C. V. Thompson

Department of Materials Science and Engineering, Massachusetts Institute of Technology, Cambridge, MA 02139

Real-time measurements of stress evolution during the deposition of VolmerWeber thin films reveal a complex interplay between mechanisms for stress generation and stress relaxation. We observed a generic stress evolution from compressive to tensile, then back to compressive stress as the film thickened, in amorphous and polycrystalline $\mathrm{Ge}$ and $\mathrm{Si}$, as well as in polycrystalline $\mathrm{Ag}, \mathrm{Al}$, and $\mathrm{Ti}$. Direct measurements of stress relaxation during growth interrupts demonstrate that the generic behavior can occur even in the absence of stress relaxation. When relaxation did occur, the mechanism depended sensitively on whether the film was continuous or discontinuous, on the process conditions, and on the film/substrate interfacial strength. For Ag films, interfacial shear dominated the early relaxation behnavior, whereas this mechanism was negligible in $\mathrm{Al}$ films due to the much stronger bonding at the $\mathrm{Al} / \mathrm{SiO}_{2}$ interface. For amorphous $\mathrm{Ge}$, selective relaxation of tensile stress was observed only at elevated temperatures, consistent with surface-diffusion-based mechanisms. In all the films studied here, stress relaxation was suppressed after the films became continuous. 


\section{INTRODUCTION}

The small length scales and reduced dimensionality associated with thin film microstructures can change the mechanical behavior of films relative to the bulk. One common example is the large residual stress often observed to develop in thin films during deposition. While in heteroepitaxial growth the primary source of stress is from lattice mismatch, most film-substrate combinations lead to Volmer-Weber (V-W) growth, without lattice matching. The microstructural stages in the V-W growth mode include (see Fig. 1): nucleation of discrete islands, island growth, island impingement and coalescence, percolation of the island array, and channel-filling to eventually form a continuous thin film, which can be polycrystalline or amorphous depending on the material and the deposition conditions. For polycrystalline thin films, an additional process, grain coarsening (grain growth) can occur during and after coalescence.

The goal of this study is to understand the detailed structural origins of thin film stress by examining stress evolution during film growth using real-time wafer curvature measurements. Previous work has shown that a complex evolution of the film stress is associated with V-W growth [1-5]. It is only now becoming widely appreciated that this behavior arises from a dynamic interplay between different stress generation mechanisms, coupled with a variety of possible mechanisms for stress relaxation. Studies of stress generation processes have focused mostly on real-time growth experiments [15], while studies of stress relaxation processes have focused on post-growth thermal cycling experiments [6-10]. Here we explore the competition between stress generation and relaxation mechanisms by combining film growth studies with isothermal growth 


\section{DISCLAIMER}

This report was prepared as an account of work sponsored by an agency of the United States Government. Neither the United States Government nor any agency thereof, nor any of their employees, make any warranty, express or implied, or assumes any legal liability or responsibility for the accuracy, completeness, or usefulness of any information, apparatus, product, or process disclosed, or represents that its use would not infringe privately owned rights. Reference herein to any specific commercial product, process, or service by trade name, trademark, manufacturer, or otherwise does not necessarily constitute or imply its endorsement, recommendation, or favoring by the United States Government or any agency thereof. The views and opinions of authors expressed herein do not necessarily state or reflect those of the United States Government or any agency thereof. 


\section{DISCLAIMER}

Portions of this document may be illegible in electronic image products. Images are produced from the best available original document. 
interrupts that further elucidate the nature of the relaxation mechanisms. We have examined a wide range of materials systems in order to obtain a comprehensive picture of stress evolution. After we present our data, a brief review of relevant mechanisms for stress generation and relaxation is given. We then discuss the implications of our data within the context of these mechanisms.

\section{EXPERIMENTS}

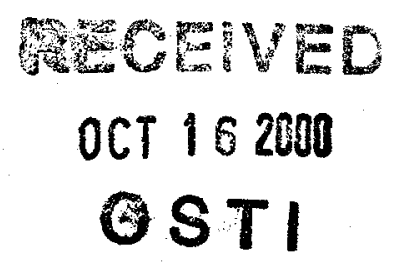

All films were grown on $\mathrm{Si}(001)$ substrates that had either a native oxide or a thermally-grown oxide. No variations in the resulting stress evolutions were observed between the two substrates. The substrates were first solvent-cleaned in an ultrasonic bath, then etched in a 4:1 $\mathrm{H}_{2} \mathrm{SO}_{4}: \mathrm{H}_{2} \mathrm{O}_{2}$ solution that reduces hydrocarbons. After transfer into the UHV chamber, the substrates were outgassed at $350^{\circ} \mathrm{C}$ for 1 hour (for metal film depositions) or $750^{\circ} \mathrm{C}$ for 5 minutes (for semiconductor film depositions).

All films were deposited by electron beam evaporation in ultra-high vacuum. Base pressures were $1 \times 10^{-10}$ Torr, while deposition pressures were in the range $5 \times 10^{-9}-3 \times 10^{-8}$ Torr, which was mostly hydrogen. Deposition rates ranged from $0.1-5 \AA / s$ and were controlled using quartz crystal monitors.

During deposition, the wafer curvature was measured using a multi-beam optical stress sensor (MOSS). MOSS is a laser deflectometer that illuminates the sample with an array of parallel laser beams, as shown in Fig. 2. Upon reflection from the substrate, divergence is introduced into the laser array due to the substrate curvature. The changing divergence is measured on a CCD camera by measuring the changes in spacing between 
adjacent laser beams. More details on the MOSS apparatus can be found elsewhere $[11,12]$.

The curvature, $\kappa$, is directly proportional to the product of the film stress and the film thickness (which we wil refer to as the "stress-thickness") [13,14]:

$$
\sigma h=\frac{M_{s} h_{s}^{2}}{6} \kappa(t)
$$

where $\sigma$ is the film stress, $h$ is the film thickness, $h_{s}$ is the substrate thickness, and $M_{s}$ is the biaxial modulus of the substrate. In a real-time growth experiment, both the stress and thickness will be time-varying. Wafer curvature must be interpreted carefully, and correlated with direct microstructural measurements, since the observed curvature depends not only on the stresses present in the film, but also on how much film material is present, how the material and stresses are spatially distributed, and how well forces are coupled between the film and substrate (i.e., on the interfacial shear strength).

Select samples were examined ex situ using atomic force microscopy (AFM) and transmission electron microscopy (TEM) in both plan-view and cross-section.

\section{RESULTS}

The curvature evolution for different V-W films grown on $\mathrm{SiO}_{2}$ are shown in Fig. 3 . Unless otherwise noted, all growths occurred at room temperature (RT). The data are plotted as stress-thickness vs thickness, where the slope of the curves represents an "incremental" stress associated both with growth of new film, and relaxation occurring 
within the existing film [12]. Note that positive values of stress-thickness imply a tensile mean stress (i.e., spatially averaged through the film), while negative values imply a compressive mean stress. Positive (negative) slopes correspond to a tensile (compressive) incremental stress, even in cases where the absolute stress-thickness is negative (positive).

Generically similar behavior was observed in all cases, with an evolution from an initial compressive stress, to tension, which reversed back into long-term compression. This behavior has been frequently observed previously [1-5]. The detailed evolution, and the magnitudes of the observed stresses, differ significantly amongst the materials used in our study. Two materials that show somewhat unique behavior are polycrystalline Ge (pGe), which exhibits a very weak tensile "peak", and $\mathrm{Ti}$, which exhibits a double-tensilepeak. Table I summarizes the maximum observed incremental stresses in the tensile and long-term compressive regimes, as well as the homologous deposition temperature, and the deposition rate.

In Fig. 4 we replot the $\mathrm{Ag}$ and $\mathrm{Al}$ curvature data, both on a thickness scale [Fig. 4(a)] and on a normalized scale that highlights the differences in behavior [Fig. 4(b)]. For Ag, there was a long initial regime over which there was no stress generation, either tensile or compressive. We obtained this result repeatedly for $\mathrm{Ag}$ on $\mathrm{SiO}_{2}$ under different deposition rates and temperatures. By comparison, $\mathrm{Al}$ films on $\mathrm{SiO}_{2}$ support stresses from the earliest stages of growth.

The circles on the curves in Fig. 4 correspond to the thicknesses where we obtained TEM microstructure data. Fig. 5 shows a side-by-side comparison of the microstructures of $\mathrm{Ag}$ and $\mathrm{Al}$ from plan-view. TEM. The TEM micrographs were acquired from graded- 
thickness samples where a shutter was moved across the wafer during deposition, in order to obtain the full range of evolution in one deposition. While evolution of the microstructure after deposition and upon exposure to air is possible, this should not affect our basic conclusions.

$\mathrm{Ag}$ islands at $120-160 \AA$ mass equivalent thickness contain numerous grain boundaries and we will refer to these as polycrystalline islands. Significant island impingement to form grain boundaries has clearly occurred prior to reaching a nominal thickness of $120 \AA$ in $\mathrm{Ag}$. Films of $\mathrm{Al}$ at $25-40 \AA$ thickness exhibit similar microstructure (although the $\mathrm{Al}$ islands have smaller height-to-width (aspect) ratios than the Ag islands). Nonetheless, the stress evolution behavior in the two materials is strikingly different. For Ag at $120 \AA$, tensile stress generation was barely starting to develop. Even at $160 \AA$ mass equivalent thickness of $\mathrm{Ag}$, near the onset of percolation, the tensile curvature has reached only $11 \%$ of its maximum value. On the other hand, for the case of $40 \AA$ thick $\mathrm{Al}$, the tensile curvature has already reached $51 \%$ of its maximum value.

Fig. 6 shows the stress evolution for amorphous Ge (a-Ge) deposited at room temperature (solid line) and at $185^{\circ} \mathrm{C}$ (dashed line). Reflection high energy electron diffraction (RHEED) patterns obtained during growth confirmed the lack of long-range order. Even for these amorphous films, the typical compressive-tensile-compressive evolution of stress associated with Volmer-Weber growth was observed. A non-contact AFM image of a $70 \AA$ thick a-Ge film deposited at $185^{\circ} \mathrm{C}$ is shown inset in Fig. 6 that confirms the V-W growth mode, and shows that the a-Ge islands have an aspect ratio of about 0.2. As shown in Fig. 6, raising the deposition temperature increased the thickness 
required to obtain the maximum in the tensile curvature (which is directly correlated with when the film becomes continuous, e.g., see Figs. 4 and 5, and [1]), and reduced the magnitudes of both the tensile and compressive stresses.

In order to examine relaxation kinetics in more detail, we performed interrupts during the deposition process, both before and after film continuity was achieved. Fig. 7 shows the behavior for a-Ge deposited at room temperature. Fig. 7(a) shows the curvature vs time, which explicitly demonstrates the relaxation kinetics, while Fig. 7(b) shows curvature vs thickness. In the latter case, the interrupts are compressed, but the plot is useful for studying the stress evolution when growth is resumed after an interrupt. Absolutely no relaxation was observed during growth interrupts at room temperature, either before or after continuity, over time scales comparable to those of the depositions themselves. Note in Fig. 7(b) that no effect from the interrupts on the stress evolution was observed when growth was resumed.

Fig. 8 shows the growth interrupt behavior for a-Ge deposited at $185^{\circ} \mathrm{C}$, again as a function of both time and thickness. Prior to film continuity a clear relaxation of the tensile stress was observed see [Fig. 8(a)]. Furthermore, the stress actually goes into net compression after about $200 \mathrm{sec}$, of annealing, as shown in detail in the inset of Fig. 8(a).

The stress relaxation due to the pre-continuity interrupt is also clearly evident in Fig. 8(b), both as a discontinuity in the stress-thickness, and as a subsequent change in slope of the stress-thickness curve. Additional film growth to full continuity resulted in a smaller, secondary tensile peak, presumably due to creation of the final grain boundaries. Another important observation is that the final incremental compressive stress is about $28 \%$ larger after the pre-continuity interrupt than for uninterrupted growth. 
During growth interrupts performed after the film became continuous, a small apparent relaxation occurred [see Fig. 8(a)]. However, this process was reversible, since the curvature eventually returned to following the same evolution curve it had prior to the interrupt. Reversible changes in stress during growth interrupts are often seen in polycrystalline metal films, and have been attributed to surface structural effects [5] rather than bulk stress relaxation. As will be shown next, we also observed large, reversible changes in stress associated with interruption and resumption of deposition in metal films. These reversible changes can make interpretation of the bulk, irreversible relaxation kinetics rather difficult. In a-Ge, however, these effects are small, allowing direct measurement of the relaxation behavior.

Fig. 9 shows the results of growth interrupts during room temperature deposition of Ag. When Ag deposition was interrupted prior to film continuity, a rapid increase in tension was observed [see inset of Fig. 9(a)]. This is the surface-related reversible change mentioned above. A slow, rather small stress relaxation was subsequently observed over the course of the interrupt in Fig. 9(a). When growth was resumed, a rapid reduction in the tensile stress took place, with a magnitude that was larger than the tensile rise at the beginning of the interrupt. Fig. 9(b) shows that the interrupt had a very large effect on stress evolution upon the resumption of growth, reducing the incremental tensile stress by $60 \%$, which directly indicates that a large degree of relaxation occurred during the interrupt. Additional growth after the interrupt resulted in a weak secondary tensile peak. The pre-continuity interrupt also reduced the long-term compressive stress, compared with the continuous deposition case. Note that this is different from a-Ge, 
where an increase in compression resulted from the pre-continuity interrupts (performed at elevated temperature).

Growth interrupts performed after the tensile peak, i.e., when the film was continuous, had little effect on the stress evolution, suggesting that no bulk stress relaxation occurred. In fact, even for films up to $2000 \AA$ thick, growth interrupts produced no observable relaxation of the compressive stress.

The results of $\mathrm{Al}$ growth interrupts are shown in Fig. 10. The pre-continuity interrupt resulted in realtively slow relaxation of the tensile stress [see Fig. 10(a)]. Note that on the time scale of the deposition process (e.g., $500 \mathrm{sec}$.), relaxation was minimal. Upon resumption of growth, Fig. 10(b) clearly shows that relaxation occurred during the first interrupt. Post-continuity interrupts for Al produced no significant stress relaxation.

An increase in the long-term compressive stress after the pre-continuity interrupt was observed in $\mathrm{Al}$, as it was for $\mathrm{a}-\mathrm{Ge}$. One caveat, however, is that it was more difficult to obtain quantitatively repeatable results for $\mathrm{Al}$ than for $\mathrm{Ag}$ or $\mathrm{Ge}$, presumably due to the highly reactive nature of the metal, which increased the sensitivity to both the process conditions and the substrate cleanliness.

In order to evaluate whether long growth interrupts created contamination layers that might promote nucleation of new islands when deposition was resumed, we grew an $\mathrm{Al}$ film at RT under typical conditions, and performed two growth interrupts, each of 600 sec. duration. A TEM cross-section micrograph of this sample is shown in Fig. 11, with the position, in depth, of the interrupts indicated on the figure. No oxide layer was observed, and the grains are fully columnar, which provides reassurance that stress 
evolution after growth interrupts was dominated by intrinsic microstructural evolution rather than by contamination effects.

Table II summarizes the results of the interrupt experiments for a-Ge, $\mathrm{Ag}$, and $\mathrm{Al}$.

\section{MECHANISMS FOR STRESS GENERATION AND RELAXATION}

We observed a compressive-tensile-compressive stress evolution behavior during Volmer-Weber growth of a variety of film materials on $\mathrm{SiO}_{2}$. The generic nature of this behavior, which has also been observed frequently by others, suggests that a few common mechanisms are responsible for the measured stress evolution. These mechanisms should not depend on the film being crystalline, since we observe similar stress evolution in amorphous Ge. The data for a-Ge will be quite important in the subsequent discussion regarding mechanisms. We note that standard V-W growth processes such as island nucleation and growth, followed by island impingement and coalescence to form a continuous film, appear to occur in a-Ge just as they do in polycrystalline films. While it is difficult to speak of grain boundaries in amorphous Ge, this network-forming material might be expected to retain regions of high local bond strain, and slightly differing density, where two independently nucleated islands coalesce.

It is also notable that we observed the compressive-tensile-compressive behavior over a wide range homologous temperatures, from $0.15-0.66$. In the work by Abermann et al., deposition of so-called "low-mobility" films (e.g., Ti) resulted only in tensile stress both before and after film continuity. However, for Ti we observe compressive stress after film continuity. Since the nominal growth conditions, including background 
pressures, are similar in Abermann's experiments and ours, the reasons for this difference are unclear.

Prior to discussing the implications of our results, it is useful to briefly review some of the mechanisms suggested in the literature for stress generation and relaxation, with an emphasis on processes occurring during island growth and coalescence. Then, in the following discussion, we examine to what extent our data are consistent with these mechanisms.

For a comprehensive discussion of intrinsic stress evolution in thin films, the reader is referred to reviews by Doerner and Nix [15], Koch [4], and Thouless [16].

\section{A. Stress Generation}

\section{Island Coalescence}

There is widespread agreement that the tensile stresses observed during the island coalescence stage of V-W film growth are associated with the formation of grain boundaries $[17,1,5,18]$. This is certainly consistent with our real-time curvature and microscopy results for $\mathrm{Ag}$ and $\mathrm{Al}$, as well as for $\mathrm{a}-\mathrm{Ge}$. One critical issue is whether the measured tensile stresses can be predicted quantitatively.

As shown schematically in Fig. 12, when two growing islands first touch, some portion of the adjacent surfaces of the islands rapidly "zip" together to form a grain boundary. Zipping, which is driven by the reduction in the overall surface energy, is accomplished through elastic deformation of the islands, thereby imposing a cost of increased strain energy. Nix and Clemens have modeled this process using a 
conceptually simple and appealing view of island zipping as a crack closure process [18]. They arrived at an expression for the mean stress due to zipping as:

$$
\sigma_{\mathrm{zip}}=\sqrt{\mathrm{E} \frac{(1+v)}{(1-v)} \frac{\Delta \gamma}{\mathrm{r}_{\mathrm{I}}}}
$$

where $\Delta \gamma=2 \gamma-\gamma_{\mathrm{gb}}$ is the energy recovered by converting two free surfaces of energy per area $\gamma$ into a grain boundary with energy per area $\gamma_{g b}, r_{I}$ is the island radius at impingement, $\mathrm{E}$ is Young's modulus, and $v$ is Poisson's ratio. Using $\mathrm{E}=100 \mathrm{GPa}, \mathrm{v}=$ $1 / 3, \Delta \gamma=2 \mathrm{~J} / \mathrm{m}^{2}$, and $\mathrm{r}_{1}=500 \AA$, equation (1) predicts a mean stress of $2.8 \mathrm{GPa}$, which is much larger than is typically observed in metals such as $\mathrm{Ag}$ or $\mathrm{Al}$ (see Table I and $[1,5]$ ).

While relaxation effects can mitigate the observed stress levels, Seel et al. have reexamined the energetics of island coalescence using finite element calculations to find the minimum in the total energy (elastic + surface) as a function of the grain boundary zipping height [19]. The Seel et al. results can be reasonably fit to a form

$$
\sigma_{z i p}=\frac{q \Delta \gamma}{\Gamma_{I}}
$$

where $\Delta \gamma$ and $r_{1}$ are the same as in Eq. (1) and $\mathrm{q}$ is a unitless constant of order 1-10. For $\mathrm{q}$ $=2.5, \Delta \gamma=2 \mathrm{~J} / \mathrm{m}^{2}$ and $\mathrm{r}=500 \AA, \sigma_{\text {zip }}=100 \mathrm{MPa}$, which is much smaller than predicted by Nix and Clemens, and is of the same order as observed in our Ag and Al experiments.

Freund has also modeled island zipping, using an analytical force-balance approach to calculate the stresses generated when a coplanar array of hemispheres impinges with 
cohesive forces [20]. His analysis, which borrows from Hertzian contact theory, also yields Eq. (2), with $q=2$.

In both the Seel and Freund models, grain boundary formation effectively creates an outward pressure on the island that generates the in-plane tensile stress [21]. The fundamental reasons for the large quantitative discrepancies between the Nix and Clemens crack closure approach, and the Seel et al. and Freund models, are not understood.

Seel et al. also examined the kinetics of tensile stress generation using a simple simulation of island nucleation, growth, coalescence, and relaxation, that incorporates their finite element results for stresses created when islands impinge [19]. The simulations reasonably reproduce the basic aspects of the tensile rise observed in $\mathrm{Ag}$ films.

\section{Capillary-induced Growth Stresses}

Another generic mechanism for stress generation in Volmer-Weber films is associated with the island growth process itself. In particular, island growth can lead to bulk stress generation due to the effects of surface stress on islands that are well adhered to the substrate $[22,23]$. These stresses can be evolving both before and during island coalescence, and will be compressive when the surface stress is tensile. Briefly, surface stress imposes a size-dependent lattice parameter on finite particles such as islands. Therefore, during island growth the lattice parameter will constantly attempt to adjust, which is only possible if the islands are laterally unconstrained by the substrate, i.e., if the shear rate at the island/substrate interface is comparable to the island growth rate. This 
may occur for very small islands in weakly interacting systems, but at large island sizes, the shear rate must eventually lag the growth rate, and the island becomes strained as a result. Defining a critical radius $\mathrm{r}_{\mathrm{LD}}$ as the size above with shear is suppressed and the island becomes "locked-down" to the substrate [24], the subsequent stress generated in a cylindrical island as a function of its radius $r$ and surface (interface) stress $f(g)$ is given approximately by [22]

$$
\sigma_{\mathrm{ig}}=\frac{(\mathrm{f}+\mathrm{g})}{\mathrm{A}}\left(\frac{1}{\mathrm{r}}-\frac{1}{\mathrm{r}_{\mathrm{LD}}}\right)
$$

where $\mathrm{A}$ is the aspect ratio (height-to-radius ratio). We are capturing here only the simplest aspects of this mechanism (which we will refer to as the "capillary-induced growth stress") in order to bring out the relevant physics. A much more detailed analysis has been given by Cammarata et al. [23]. From Eq. (3), the asymptotic stress will be of order $-(f+g) / r_{L D}$. For $A=1, f+g=2 J / m^{2}$ and $r_{L D}=10-100 \AA$, compressive stresses of order 100-1000 MPa are predicted, which are comparable to experimental values (see Table I). Note that whereas zipping stresses become constant after the film is continuous, capillary-induced growth stresses will continue to evolve as the film thickens due to the effects of the free surface and film/substrate interface on the in-plane lattice parameter of the continuous film [23].

\section{Superposition}

Fig. 13 schematically illustrates how the mean zipping stress and capillary-induced growth stress might evolve during deposition. The combined effects on curvature 
evolution are also shown. In the drawing, we have assumed that the asymptotic value of the capillary-induced growth stress has opposite sign and larger magnitude than that due to island zipping. From Eqs. (2) and (3), taking $(f+g) /(A \Delta \gamma)=1$ and $q=2$, capillaryinduced growth stress will exceed the zipping stress when $r_{L D}<0.5 r_{I}$ (i.e., on average islands must lock down well before impingement), which should be satisfied under many conditions of nucleation and growth [25]. Clearly, the detailed behavior will depend both on the absolute values of the surface stresses and energies, and on the kinetics of nucleation and growth, but the important point is that the compressive-tensilecompressive behavior can result naturally from the mechanisms discussed above, even without relaxation effects included. Additionally, in this view, the early- and late-stage compressive behavior results from the same mechanism, and the prominent tensile peak often observed is primarily a consequence of the rapid increase in grain boundary area associated with the percolation stage of growth, and need not imply that zipping stresses are much larger in magnitude than capillary-induced growth stresses.

\section{Other Mechanisms}

There are a variety of other possible mechanisms for generating stress. Notable amongst these are direct action of surface stress, interstitial incorporation, grain growth, and film-substrate reactions.

Spaepen has analyzed the direct action of surface stresses of liquid drops [26] and solid islands [27] on substrates. In this case, a true bulk stress is not generated within the film, but a substrate curvature does result from the balance of forces at the surface. However, in this case the curvature varies as $\mathrm{f} / \mathrm{h}_{\mathrm{s}}{ }^{3}$, whereas curvature due to the capillary- 
induced growth stress model above varies as $f /\left(\mathrm{rh}_{\mathrm{s}}{ }^{2}\right)$ [28], where $h_{\mathrm{s}}$ is the substrate thickness. The latter is expected to be much larger than the former.

It has also been suggested that extra atoms may be incorporated into the film as interstitials during growth, thereby explaining the long-term compressive stress observed after the film becomes continuous [28]. The propensity towards interstitial incorporation results from the tendency of tensile surface stresses to splay step edges. This kinetic trapping process should rapidly become more important as homologous deposition temperature is reduced, both due to the exponentially increasing trapping probability, and due to decreasing relaxation rates. However, it is not clear that this is borne out in experiments $[29,30]$.

Grain growth may also affect stress evolution. Careful examination of our TEM data in Fig. 5 clearly shows that grain growth is occurring even during the island coalescence process, and probably within our continuous films as well (although this has not been directly verified yet). Unfortunately, the overall effects of grain growth on stress are difficult to specify. Grain growth may generate stresses if grain boundaries have densities that differ from the bulk [31]. However, grain growth can also relax stresses, either due to the removal of boundary segments formed by zipping, or due to preferential growth of elastically soft or plastically soft grains [32-35]. While some evidence for $\cdot$ grain growth effects on stress have been inferred from thermal cycling experiments (e.g., see [6]), we note that growth interrupts of continuous Ag films, both in this work and in the work of others $[1,5]$, produce no changes in the bulk film stress, even though grain growth is likely to be occurring [1]. 
Finally, if a film reacts with the substrate during deposition, with an accompanying change of density in the new phase, stress will result. This may be an important contribution to the stress evolution of $\mathrm{Ti}$ and $\mathrm{Al}$, as will be discussed later.

\section{B. Stress Relaxation}

Stress relaxation processes can occur during V-W film growth in response to stress generation. Relaxation will affect both the shape of the stress evolution curve, as well as the magnitude of the observed stresses. To complicate matters, relaxation kinetics are strongly dependent on deposition temperature and on microstructural length scales. Several mechanisms for stress relaxation are of interest here.

\section{Interfacial shear}

The ability of a thin film to support stress must depend sensitively on the strength of the film/substrate interface. Interfacial shear will be significant when films are discontinuous (so that resolved shear stresses are present at the island perimeters) and weakly bound to their substrates. When the island array percolates to form a continuous network, interfacial shear will typically become suppressed, since it must now occur over lateral dimensions of order the wafer size. Also, in fully continuous films the stress state will be largely biaxial, with no resolved shear stresses at the film/substrate interface to drive the shear process. To our knowledge, the atomistic nature of shear occurring at a crystal/amorphous interface (e.g., metal films on $\mathrm{SiO}_{2}$ ) has not been elucidated. We use 
the term "interfacial shear" instead of "interfacial sliding" that has been previously used in order to suggest the passage of dislocation-like entities at the film/substrate interface.

Interfacial shear tend to relax all stresses, both the compressive capillary-induced growth stress, and the tensile stress due to grain boundary zipping [19].

2. Inclined shear

Passage of dislocations on glide planes inclined relative to the film/substrate interface can also occur, in analogy with the propagation of $60^{\circ}$ misfit dislocations in cubic heteroepitaxial systems $[6,16]$. The energetics and kinetics of inclined shear in typical polycrystalline thin films will be determined by grain size, film thickness, grain crystallographic orientation, the density and strength of dislocation pinning sites, the presence/nature of a passivating surface layer, and temperature. Inclined shear will tend to relax all stresses present in the film, both tensile and compressive, and can operate in both discontinuous and continuous films.

\section{Viscous flow}

In amorphous films, viscous flow can relieve both tensile and compressive stresses within islands or continuous films.

\section{Surface diffusion}

In the early stages of island growth, significant morphological rearrangement can take place via surface diffusion. This can occur via classical Ostwald ripening of discrete islands, or it can occur in coalesced islands [36]. In the latter case, when two different size islands impinge to form a grain boundary, the smaller island can "dissolve" onto the 
surface of the larger island [37]. Morphological rearrangement of this sort will relax the tensile stress that was created upon grain boundary formation. However, morphological rearrangement cannot, by itself, relax the compressive stresses associated with surface stress effects during island growth.

Alternatively, the tensile stresses generated by grain boundary formation can be relieved by diffusion of adatoms on the free surface of a film, followed by diffusion down into the grain boundary $[9,10]$, as illustrated in Fig. 12(c). This serial process bears analogy with Coble creep in bulk polycrystals. "Surface-Coble" creep can relieve all the tensile stress created by grain boundary zipping. For grain aspect ratios greater than about 3:1, creep will not relieve other stresses (such as capillary-induced growth stress) within the central region of the grain, unless some interfacial shear also takes place [38].

\section{DISCUSSION}

In this section we will examine our stress evolution data within the context of the competition between stress generation due to island growth and coalescence, with particular emphasis on the amorphous and polycrystalline Ge data. Then we will comment on the role of film/substrate reactions in stress generation. Finally, implications of the data regarding specific mechanisms for relaxation are discussed.

Our stress evolution results for a-Ge provides compelling support for the picture of two simultaneous and competing mechanisms for stress generation leading to the generic compressive-tensile-compressive behavior. For a-Ge at RT, growth interrupts directly show that no relaxation occurred at any stage of growth (see Fig. 7). Despite this, the 
turnover from tensile stress to compressive stress was still clearly observed. This implies that the long-term compressive stress, which we attribute to surface stress effects occurring during island growth, has a larger magnitude than the final tensile stress due to island coalescence, as discussed earlier. Similar reasoning applies to the case of $\mathrm{Al}$ deposited at room temperatuie, where negligible stress relaxation occurred on the time scale of the uninterrupted growth experiments.

The behavior of a-Ge deposited at elevated temperature provides additional evidence that the long-term compressive stress actually arose in the early stages of growth. In particular, growth interrupts prior to film continuity show that the film can relax from tension into net compression, presumably as local stresses near coalescence boundaries were preferentially relieved, thereby "unmasking" the co-existing compressive stress in the island interiors (see Fig. 8). The observation that resumption of growth after the precontinuity interrupt leads to a significantly higher apparent compressive stress in the continuous films also supports this hypothesis. More will be said about the relaxation mechanisms momentarily; however, we note now that the small aspect ratio of the islands is consistent with being able to retain some stress within the interior of a coalesced island while relaxing localized stresses at the coalescence boundaries themselves.

For a-Ge deposited at elevated temperature, we also observed that the tensile peak occurred later, and the magnitudes of the tensile and compressive stresses were lower, than for room temperature deposition (see Fig. 6). Although the retarded stress evolution must in part be due to increasing rates for thermally activated relaxation processes, the dominant effect more likely arises from the coarser length scales for island nucleation, which tends to increase the thickness required for film continuity, and suppresses the 
absolute stress magnitudes even in the absence of relaxation. Similar temperaturedependent behavior has been observed in Ag films and simulated in detail by Seel et al [19]. The observation that the tensile peak occurs at larger thickness in the interrupted films also suggests that some coarsening occurred during the interrupt. This is true in $\mathrm{Ag}$ and Al films as well.

Additional support for the view that the compressive-tensile-compressive behavior results primarily from a competition between zipping vs capillary-induced growth stresses is provided by the experimental results for polycrystalline $\mathrm{Ge}$, which exhibits a weak tensile peak superimposed on a continuously evolving compressive stress (Fig. 3). In this case the early and late-stage compression clearly seem to arise from the same mechanism. The limited tensile rise in this case is presumably due to efficient relaxation of the tensile component via surface-diffusion-based mechanisms, which will be discussed in more detail below.

The magnitudes of the tensile stresses that we observed are generally an order of magnitude smaller than those predicted by the Nix-Clemens crack closure model of grain boundary zipping [18], with the possible exception of $\mathrm{Ti}$ (see Table I). This is true even in the case of a-Ge at room temperature, where relaxation was fully suppressed. Although this might cast some doubt on the validity of the crack closure approach, to be fair it must be emphasized that, if the observed stresses arise as a result of a superposition of simultaneously acting mechanisms for tensile and compressive stress generation, then we are only observing the stress difference between the two mechanisms at any given time. The actual magnitudes of the individual stress components might be much larger, 
but unfortunately this cannot be extracted from the experimental curvature data without additional information.

Ti films exhibit an unusual double-peak structure in the stress evolution (see Fig. 3). We believe that this results from partial reduction of the native silicon oxide to form $\mathrm{TiO}_{\mathrm{x}}$ : Recently, Dunn et al. performed Ti deposition on $\mathrm{SiO}_{2}$ in situ in a UHV-TEM [39]. They observe an initial nucleation and growth stage characterized by a specific texture, followed by a secondary island nucleation stage corresponding to a change in the preferred texture. Dunn et al. proposed that Ti partially decomposes the $\mathrm{SiO}_{2}$ to form $\mathrm{TiO}_{\mathrm{x}}$ in the first 10-20 $\AA$ of Ti deposition. Subsequently, additional Ti deposition leads to nucleation of pure $\mathrm{Ti}$ islands on the $\mathrm{TiO}_{\mathrm{x}}$ layer. This could explain our stress evolution data, with the first tensile peak corresponding to the growth of the $\mathrm{TiO}_{\mathrm{x}}$ reaction layer, and the second peak corresponding to the $\mathrm{V}-\mathrm{W}$ growth of pure $\mathrm{Ti}$ on $\mathrm{TiO}_{\mathrm{x}}$. It is unknown to what extent the large tensile stress (1.8 GPa, see Table I) was determined by the oxidation-reduction process vs island zipping process associated with $\mathrm{V}-\mathrm{W} \mathrm{TiO}_{\mathrm{x}}$ growth.

Poppeller and Abermann have reported results consistent with this picture [40]. They showed directly that deposition of clean $\mathrm{Ti}$ on pre-deposited $\mathrm{TiO}_{\mathrm{x}}$ layers. leads to the typical compressive-tensile-compressive curve that they attribute to V-W growth of $\mathrm{Ti}$ islands on the $\mathrm{TiO}_{\mathrm{x}}$. However, it must be noted that these growths took place at slightly elevated temperatures $\left(105-130^{\circ} \mathrm{C}\right)$. At room temperature, Schneewei $\beta$ and Abermann find tensile stress [41] during deposition of $\mathrm{Ti}$ on $\mathrm{MgF}_{2}$ coated substrates, with no postcontinuity compression, which is different from what we see. Unfortunately, Schneewei $\beta$ and Abermann did not report on stress evolution for $\mathrm{Ti}$ on $\mathrm{TiO}_{\mathrm{x}}$ at room temperature. 
Interaction with the substrate may also play a role in the very early stages of $\mathrm{Al}$ deposition. It is observed in Fig. 4 that tensile stress generation in $\mathrm{Al}$ occurred immediately upon deposition, and that the rate of tensile stress generation did not peak during percolation, where the rate of grain boundary formation was the highest (as is the case for $\mathrm{Ag}$ ), but instead continuously decreased during coalescence. Similar to the $\mathrm{Ti}$ case, the $\mathrm{Al}$ islands may partially react with the $\mathrm{SiO}_{2}$ substrate to form $\mathrm{AlO}_{x}$, thereby generating additional tensile stress in the earliest stages of growth.

Our data for a-Ge, $\mathrm{Ag}$, and $\mathrm{Al}$ also provides insight into the nature of stress relaxation processes occurring in both continuous and discontinuous films. In all the films studied here, no relaxation was observed during interrupts after the films became continuous. This immediately implies that viscous flow (in the case of a-Ge) and inclined shear (in the case of $\mathrm{Ag}, \mathrm{Al}, \mathrm{Ti}, \mathrm{p}-\mathrm{Si}$, and $\mathrm{p}-\mathrm{Ge}$ ) did not occur under the time-temperature conditions used in our experiments. It is important to reemphasize that the rapid tensile rise observed in the metal films when deposition was interrupted arises from surface phenomena, and this process was fully reversed upon resumption of growth [5]. In all cases, we find that the stress evolution when growth was resumed after a post-continuity interrupt proceeds with identical behavior to the evolution just prior to the interrupt. Therefore, no relaxation of bulk stress could have occurred, as this would lead to a discontinuity in the curvature (when plotted vs thickness scale), as well as a change in slope. For our purposes, the primary consequence of the surface-related reversible tensile rise will be to complicate the analysis of stress relaxation in discontinuous films of $\mathrm{Ag}$.

Fortunately, in a-Ge, this surface effect is quite small, and we can examine the precontinuity relaxation in detail. While at room temperature all relaxation processes were 
quenched out, at elevated temperature we found that tensile-stressed films relaxed during pre-continuity interrupts into a state of net compression. That is, there appears to be preferential relaxation of the localized tensile stresses generated due to island coalescence, while preserving at least some of the compressive stress due to island growth. Of the relaxation mechanisms described earlier, this behavior is consistent only with surface-diffusion-based relaxation, since all the other mechanisms will tend to relax both the tensile and compressive components. It is not possible for us to specify definitively whether morphological rearrangements such as coarsening, or surface-Coble creep, dominate the relaxation behavior, although some coarsening is clearly occurring. The lack of relaxation in continuous films points towards the coarsening mechanism, since creep would be expected to continue after continuity.

Surface diffusion is likely to be responsible for the very weak tensile peak observed during island coalescence in p-Ge. Non-contact atomic force microscopy of p-Ge shows that, even at $1200 \AA$ total thickness, the film is not fully continuous, consistent with rapid coalescence coarsening at the growth temperature of $530^{\circ} \mathrm{C}$.

While surface-diffusion-based mechanisms dominate in stress relaxation of discontinuous Ge films (both amorphous and crystalline), another mechanism must have contributed to the relaxation of discontinuous $\mathrm{Ag}$ films on $\mathrm{SiO}_{2}$. During the early stages of growth for $\mathrm{Ag}$ and $\mathrm{Al}$, there was a striking difference in the stress evolution, as shown in Figs. 4 and 5. Ag films only began supporting stress at island percolation, even though copious grain boundary formation clearly had occurred prior to this. By comparison, $\mathrm{Al}$ films supported tensile stress from the earliest stages of island growth. Abermann observed similar behavior for $\mathrm{Ag}$ and $\mathrm{Al}$ on $\mathrm{MgF}_{2}$ substrates [2]. The apparent inability 
of Ag islands to support stress immediately suggests that shear is occurring at the $\mathrm{Ag} / \mathrm{SiO}_{2}$ interface. Interfacial shear will be much more efficient in weakly bound film/substrate systems and we expect that the $\mathrm{Ag} / \mathrm{SiO}_{2}$ bonding is much weaker than $\mathrm{Al} / \mathrm{SiO}_{2}$, given the much stronger oxidation potential of $\mathrm{Al}$. We confirmed this through a series of simple stud-pull adhesion tests on identical $\mathrm{Ag}$ and $\mathrm{Al}$ films on oxidized $\mathrm{Si}$ substrates. The Ag films delaminated from the substrates at an average force of $3.3 \mathrm{lbs}$. By contrast, the Al films failed to delaminate from the $\mathrm{SiO}_{2}$ until the $\mathrm{Si}$ wafer shattered [42]. Abermann and Koch have also suggested that $\mathrm{Ag}$ "sliding" may occur on $\mathrm{MgF}_{2}$ [1].

The growth interrupt experiments provide further information on the nature of relaxation. Analysis of the time-dependence of stress relaxation during the pre-continuity growth interrupt in $\mathrm{Ag}$ is complicated by the significant tensile rise associated with surface effects [see Fig. 9(a)]. In particular, the apparent relaxation (as defined in Fig. 14) is small, which is inconsistent with the large degree of relaxation implied in Fig. 9(b) when growth is resumed after the interrupt. Since the rapid tensile rise during interrupts has been demonstrated to be reversible [5], we can account for it in a simple manner as shown in Fig. 14. The stress-thickness at the instant growth was interrupted is indicated by the dot. Any reversible process must return the curvature to this value. Therefore, the true, irreversible bulk stress relaxation that we are interested in will be given by the distance between the dot and the curvature value upon resumption of growth, which we estimate by extrapolating back the subsequent tensile rise, as shown in the figure. The degree of relaxation determined in this fashion is $62 \%$, which is in good agreement with the $60 \%$ degree of relaxation obtained by comparing the slopes before and after interrupt in Fig. 9(b). 
Fig. 9(b) demonstrates that the pre-continuity growth interrupt in Ag reduced both the tensile and long-term compressive stresses. This is certainly consistent with the interfacial shear mechanism and cannot be explained by solely by coalescence coarsening or surface-Coble creep (although there may be some contribution to the overall relaxation from these mechanisms, since coalescence is believed to be occurring and since surfaceCoble creep has been observed in $\mathrm{Ag}$ films at room temperature [9]).

For $\mathrm{Al}$, the relaxation kinetics during growth interrupts are clearly much slower than for Ag [see Fig. 10(a)]. Over 2300 sec., a 33\% relaxation was observed, compared to a $60 \%$ relaxation over $700 \mathrm{sec}$. for Ag. Again, this is consistent with a strong dependence of interfacial shear rates on film/substrate bond strength. Surface-diffusion might also play a role in the slow relaxation of Al. Mechanisms such as surface-Coble creep should relax only the stresses near the grain boundaries. TEM and AFM measurements indicate that $\mathrm{Al}$ grains have at least a 1:5 aspect ratio. Thus, as with a-Ge, surface-diffusion-based relaxation should result in greater residual long-term compression after a pre-continuity interrupt, which we do seem to observe in Fig. 10(b).

\section{CONCLUSIONS}

We have explored the detailed evolution of stress using real-time wafer curvature measurements during UHV deposition and growth interrupts of Volmer-Weber films on $\mathrm{SiO}_{2}$. Generically similar compressive-tensile-compressive behavior was observed over a range of materials, lattice structures, and homologous temperatures that is consistent with 
a competition between stress generation mechanisms due to island zipping and island growth, mediated by simultaneous relaxation mechanisms whose nature depends on the material. In a-Ge that was highly quenched, no relaxation occurred during growth interrupts at different stages of the V-W process. Nonetheless, a-Ge still exhibits the classic compressive-tensile-compressive behavior, which supports the notion of simultaneously competing stress generation mechanisms, and which further implies that the compressive stress component has a larger steady-state magnitude than the tensile component. At higher temperatures, relaxation of discontinuous a-Ge films was observed that both indicates the co-existence of compressive and tensile stress components, and is consistent with surface-diffusion-based relaxation mechanisms. At even higher temperatures, $\mathrm{p}$-Ge forms, with only a small tensile peak superimposed on a clear compressive trend, which lends further credence to the view that early and late stage compression arises from the same mechanism.

Comparison of the stress evolution for $\mathrm{Ag}$ and $\mathrm{Al}$ growth on $\mathrm{SiO}_{2}$ indicates that prepercolated Ag films do not support stress while Al films clearly do, even though the microstructures are similar. We attribute this primarily to shear occurring at the $\mathrm{Ag} / \mathrm{SiO}$ interface, which is suppressed in the much stronger $\mathrm{Al} / \mathrm{SiO}_{2}$ interface. There are also indications that surface-Coble creep may play a role in $\mathrm{Ag}$ and $\mathrm{Al}$ relaxation, but our data are far from definitive in this case.

In all the films studied here, growth interrupts after the films became continuous exhibit no measurable relaxation, indicating that processes such as inclined shear and viscous flow are not occurring at these temperatures and stress levels. 
Finally, we reiterate that in V-W film growth, the observed stress evolution results from a superposition of effects arising from multiple stress generation mechanisms, combined with a competition between multiple relaxation pathways. Further quantitative progress in this area will require that we isolate just one mechanism, which will likely require either lithographic approaches, or detailed microscopic observation in situ.

\section{ACKNOWLEDGMENTS}

We are happy to acknowledge useful discussions with Ben Freund, Allan Bower, Robert Cammarata, David Srolovitz, Frans Spaepen, Phillip Duxbury, David Jesson and Karl Sieradzki. Our thanks to Heidi Ruffner and Joe Sobczak for the pull test measurements. E. C. acknowledges support from the MRSEC at Brown University. Sandia is a multiprogram laboratory operated by Sandia Corporation; a Lockheed Martin Company, for the United States Department of Energy under Contract DE-ACO494AL85000. 


\section{References:}

1. R. Abermann and R. Koch, Thin Solid Films 129, 71 (1985).

2. R. Abermann, Vacuum 41, 1279 (1990).

3. D. Winau, R. Koch, A. Fuhrmann, and K. H. Rieder, J. Appl. Phys. 70, 3081 (1991).

4. R. Koch, J. Phys.: Condens. Matter 6, 9519 (1994).

5. A. L. Shull and F. Spaepen, J. Appl. Phys. 80, 6243 (1996).

6. W. D. Nix, Met. Trans. A 20A, 2217 (1989).

7. W. D. Nix, Scripta Mater. 39, 545 (1998).

8. M. D. Thouless, J. Gupta, and J. M. E. Harper, J. Mater. Res. 8, 1845 (1993).

9. M. D. Thouless, Acta Metall. Mater. 41, 1057 (1993).

10. M. Kobrinsky and C. V. Thompson, Appl. Phys. Lett. 73, 2429 (1998).

11. J. A. Floro, E. Chason, and S. R. Lee, Mat. Res. Soc. Symp. Proc. 406, 491 (1996).

12. J. A. Floro, E. Chason, S. R. Lee, R. D. Twesten, and R. Q. Hwang, J. Elec. Mater. 26, 983 (1997).

13. G. G. Stoney, G. G. Proc. Roy. Soc. A82, 172 (1909).

14. Flinn, P. A., Gardner, D. S., and Nix, W. D. IEEE Trans. Elec. Dev. ED-34, 6 (1987). 
15. M. F. Doerner and W. D. Nix, CRC Crit. Rev. in Sol. State and Mat. Sci. 14, 225 (1988).

16. M. D. Thouless, Annu. Rev. Mater. Sci. 25, 69 (1995).

17. R. W. Hoffman, Thin Solid Films, 34185 (1976).

18. W. D. Nix and B. M. Clemens, J. Mater. Res. 14, 3467 (1999).

19. S. C. Seel, C. V. Thompson, S. J. Hearne, and J. A. Floro, J. Appl. Phys., in press.

20. L. B. Freund, private communication.

21. R. C. Cammarata, private communication.

22. R. C. Cammarata, Progress in Surf. Sci. 46, 1 (1994).

23. R. C. Cammarata, T. M. Trimble, and D. J. Srolovitz, J. Mater. Res. in press.

24. Defining a single critical size is somewhat artificial, but theoretically convenient

25. C. V. Thompson, J. Mater. Res. 14, 3164 (1999).

26. F. Spaepen, J. Mech. Phys. Sol. 44, 675 (1996).

27. F. Spaepen, Acta Mater. 48, 31 (2000).

28. Spaepen's derivation for the curvature imposed by a drop assumes that the drop size is larger than the substrate thickness, which does not seem to be a case relevant to. most thin films. 
29. G. Thurner and R. Abermann, Thin Solid Films 192, 277 (1990).

30. Deposition of $\mathrm{Ag}$ and $\mathrm{Ti}$ films near $-100^{\circ} \mathrm{C}$ leads only to tensile stress rather than increased compression. J. A. Floro, S, J. Hearne, J. A. Hunter, and P. Kotula, E. Chason, S. C. Seel and C. V. Thompson, to be published elsewhere.

31. P. Chaudhari, J. Vac. Sci. Tech. 9,520 (1972).

32. J. A. Floro, C. V. Thompson, R. Carel and P. D. Bristowe, J. Mater. Res. 9, 2411 (1994).

33. E. M. Zielinski, R. P. Vinci, and J. C. Bravman, Appl. Phys. Lett. 67, 1078 (1995).

34. E. M. Zielinski, R. P. Vinci, and J. C. Bravman, J. Appl. Phys. 76, 4516 (1994).

35. J. E. Sanchez and E. Arzt, Scripta Metall. Mater. 27, 285 (1992).

36. J. A. Floro, M. B. Sinclair, E. Chason, L. B. Freund, R. D. Twesten, R. Q. Hwang, and G. A. Lucadamo, Phys. Rev. Lett. 84, 701 (2000).

37. F. Family and P. Meakin, Phys. Rev. Lett. 61, 428 (1988).

38. H. Gao, L. Zhang, W. D. Nix, C. V. Thompson, and E. Arzt, Acta Mater. 47, (2865 1999).

39. D. N. Dunn, R. Hull, F. M. Ross, R. M. Tromp, submitted to J. Appl. Phys.

40. M. Poppeler and R. Abermann, Thin Solid Films 295, 60 (1997).

41. H. J. Schneewei $\beta$ and R. Abermann, Vaccum 43, 463 (1992). 
42. The studs were epoxy bonded to the films and cured at $150^{\circ} \mathrm{C}$, so that the adhesion is not necessarily characteristic of the as-deposited films at RT. However, we believe that the conclusion that $\mathrm{Al} / \mathrm{SiO}_{2}$ has a much higher interfacial strength than $\mathrm{Ag} / \mathrm{SiO}_{2}$ is still strongly supported by the pull test results. 
Table I. Observed Incremental Stresses During V-W Growth

\begin{tabular}{|c|c|c|c|c|}
\hline Material & $\begin{array}{c}\text { Maximum } \\
\text { Tensile Stress } \\
(\mathbf{G P a})\end{array}$ & $\begin{array}{c}\text { Maximum } \\
\text { Compressive } \\
\text { Stress (GPa) }\end{array}$ & $\begin{array}{c}\text { Homologous } \\
\text { Deposition } \\
\text { Temperature }\end{array}$ & $\begin{array}{c}\text { Deposition } \\
\text { Rate } \\
(\AA / \mathbf{s})\end{array}$ \\
\hline $\mathbf{A g}$ & 0.19 & -0.06 & 0.24 & 2 \\
\hline $\mathbf{A l}$ & 0.12 & -0.13 & 0.32 & 2 \\
\hline $\mathbf{T i}$ & 1.8 & -0.20 & 0.15 & 2 \\
\hline $\mathbf{p - S i}$ & 0.38 & -0.25 & 0.46 & 1 \\
\hline $\mathbf{p - G e}$ & 0.02 & $-0.13 /-0.05^{*}$ & 0.66 & 1 \\
\hline $\mathbf{a - G e}$ & 0.32 & -0.50 & 0.25 & 1 \\
\hline $\mathbf{a - G e}$ & 0.06 & -0.20 & 0.35 & 1 \\
\hline
\end{tabular}

*early/late stage compressive stress

Table II. Effect of Growth Interrupts on Stress Relaxation

\begin{tabular}{|c|l|l|l|}
\hline $\begin{array}{c}\text { Material } \\
\text { (deposition temp.) }\end{array}$ & $\begin{array}{c}\text { PRE-continuity } \\
\text { interrupt* }\end{array}$ & $\begin{array}{c}\text { POST-continuity } \\
\text { interrupt* }\end{array}$ & \multicolumn{1}{|c|}{$\begin{array}{c}\text { Final compressive } \\
\text { stress }\end{array}$} \\
\hline $\begin{array}{c}\text { a-Ge } \\
(\mathbf{R T})\end{array}$ & No relaxation & No relaxation & $\begin{array}{l}\text { No effect due to } \\
\text { interrupts }\end{array}$ \\
\hline $\begin{array}{c}\text { a-Ge } \\
\left(\mathbf{1 8 5} \mathbf{~}^{\circ} \mathbf{C}\right)\end{array}$ & $\begin{array}{l}\text { Relaxed into net } \\
\text { compression }\end{array}$ & No relaxation & $\begin{array}{l}\text { Increased after PRE- } \\
\text { continuity interrupt }\end{array}$ \\
\hline $\begin{array}{c}\mathbf{A g} \\
(\mathbf{R T})\end{array}$ & $\begin{array}{l}\text { Rapid relaxation } \\
\text { towards zero stress }\end{array}$ & No relaxation & $\begin{array}{l}\text { Decreased after PRE- } \\
\text { continuity interrupt }\end{array}$ \\
\hline $\mathbf{A l}$ & $\begin{array}{l}\text { Sluggish relaxation } \\
\text { towards zero stress }\end{array}$ & No relaxation & $\begin{array}{l}\text { Increased after PRE- } \\
\text { continuity interrupt? }\end{array}$ \\
\hline
\end{tabular}

* Relaxation of bulk stresses, after accounting for any reversible surface-related changes in curvature. 


\section{Figure Captions}

Figure 1. Microstructural evolution of thin films growing in the Volmer-Weber mode.

Figure 2. The MOSS setup for measuring wafer curvature in real-time.

Figure 3. Stress-thickness vs thickness during deposition of $\mathrm{Ag}, \mathrm{Al}, \mathrm{Ti}$, polycrystalline $\mathrm{Si}$, polycrystalline $\mathrm{Ge}$, and amorphous $\mathrm{Ge}$.

Figure 4. Direct comparison of curvature evolution for $\mathrm{Al}$ (dash line) and $\mathrm{Ag}$ (solid line) on $\mathrm{SiO}_{2}(\mathrm{RT}, 2 \AA / \mathrm{s})$. (a) Stress-thickness vs thickness. (b) Scales have been normalized by the values at the tensile peak of each curve in (a). The circles in (a) correspond to where TEM micrographs are displayed in Fig. 5.

Figure 5. Plan-view TEM micrographs of $\mathrm{Ag}$ films (upper panels) and $\mathrm{Al}$ films (lower panels) on $\mathrm{SiO}_{2}$.

Figure 6. Stress-thickness vs thickness for a-Ge films grown at RT (solid line) and $185^{\circ} \mathrm{C}$ (dots). An AFM image from a $70 \AA$ film grown at $185^{\circ} \mathrm{C}$ is shown inset. The sides of the image are $2120 \AA$ across.

Figure 7. Growth interrupt behavior for a-Ge grown at RT. (a) Stress-thickness vs time, and (b) stress-thickness vs thickness. The solid line is for growth with interrupts, while the dots are uninterrupted growth data. The pre-continuity interrupt behavior is magnified in the inset shown in (a). The arrows in (b) indicate the thickness at which the interrupts occurred. The "shoulder" on the solid-line data at $70 \AA$ in (b) is due to a data acquisition glitch.

Figure 8. Growth interrupt behavior for a-Ge grown at $185^{\circ} \mathrm{C}$. (a) . Stress-thickness vs time, and (b) stress-thickness vs thickness. The solid line is for growth with interrupts, while the dots are uninterrupted growth data. The relaxation during the pre-continuity 
interrupt is magnified in the inset shown in (a), where the arrow indicates when the interrupt started. The arrows in (b) indicate the thickness at which the interrupts occurred.

Figure 9. Growth interrupt behavior for Ag films. (a) Stress-thickness vs time, and (b) stress-thickness vs thickness. The solid line is for growth with interrupts, while the dots are uninterrupted growth data. The pre-continuity interrupt behavior is magnified in the inset shown in (a). The tensile rise above the dashed line in the inset occurs due to reversible surface effects. The triangles in (b) show where the incremental tensile and compressive stresses were determined.

Figure 10. Growth interrupt behavior for $\mathrm{Al}$ films (a). Stress-thickness vs time, and (b) stress-thickness vs thickness. The solid line is for growth with a pre-continuity interrupt, while the dots are data for growth with a post-continuity interrupt. The behavior immediately after the interrupts begin is magnified in the inset shown in (a), where the arrows indicate the start of the interrupts.

Figure 11. Cross-section TEM micrograph of an Al film growth with two $600 \mathrm{sec}$. interrupts during deposition. (a) Bright field image, and (b) oxygen map. The positions of the interrupts are indicated, but no oxide layer or lattice disruption is observed at these locations.

Figure 12. Tensile stress generation by island impingement. (a) Islands are just touching. (b) Islands rapidly deform towards one another, "zipping" together to form a grain boundary and creating tensile stress. The shaded region is formed entirely via elastic deformation, not mass transport. (c) Surface-Coble creep: surface diffusion of adatoms followed by diffusion down into the grain boundary to "fill in" the shaded region relaxes all the tensile stress. Relaxation due to inclined shear and interfacial shear are also schematically indicated.

Figure 13. (a) Schematic evolution of the effective stress due to grain boundary zipping and island growth. The solid curve is the sum of the previous two, representing the 
overall effective stress. (b) The overall stress-thickness evolution, obtained by multiplying the solid curve in (a) by the film thickness.

Figure 14. Stress-thickness evolution during the pre-continuity interrupt of Ag, which is a superposition of reversible surface effects and bulk stress relaxation. The true degree of bulk relaxation is defined as the difference between the stress-thickness at the point where growth was interrupted, and the stress-thickness when growth is resumed. The latter is estimated by extrapolating back from the second tensile peak. The apparent relaxation is also shown. 


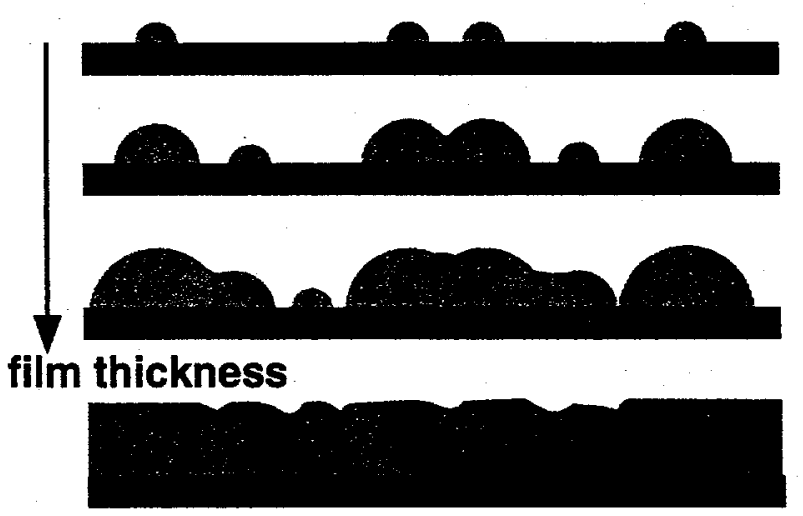

Figure 1

J. A. Floro 


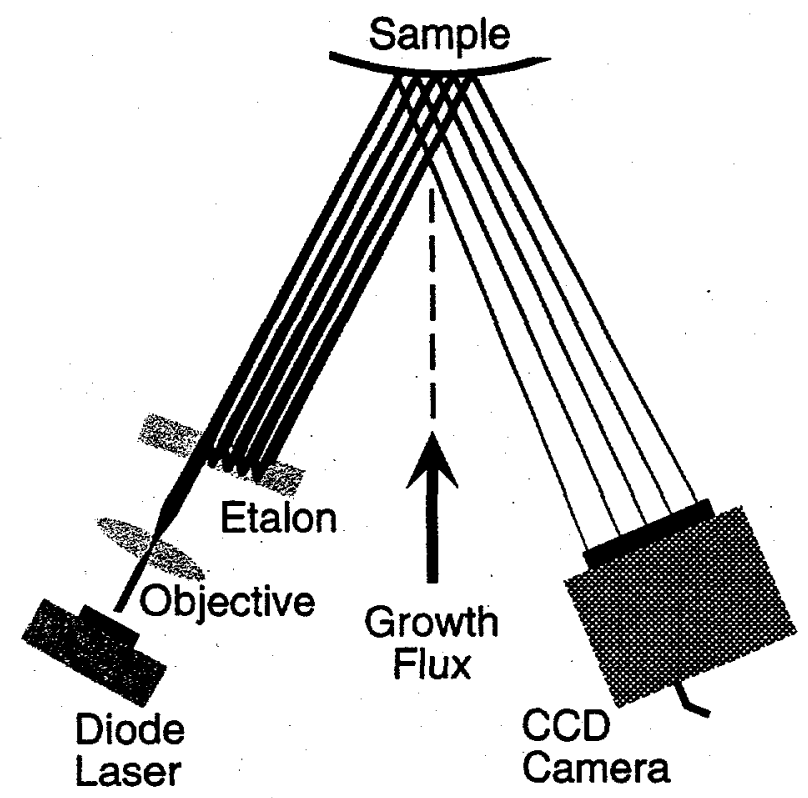

Figure 2

J. A. Floro 


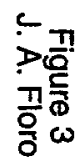

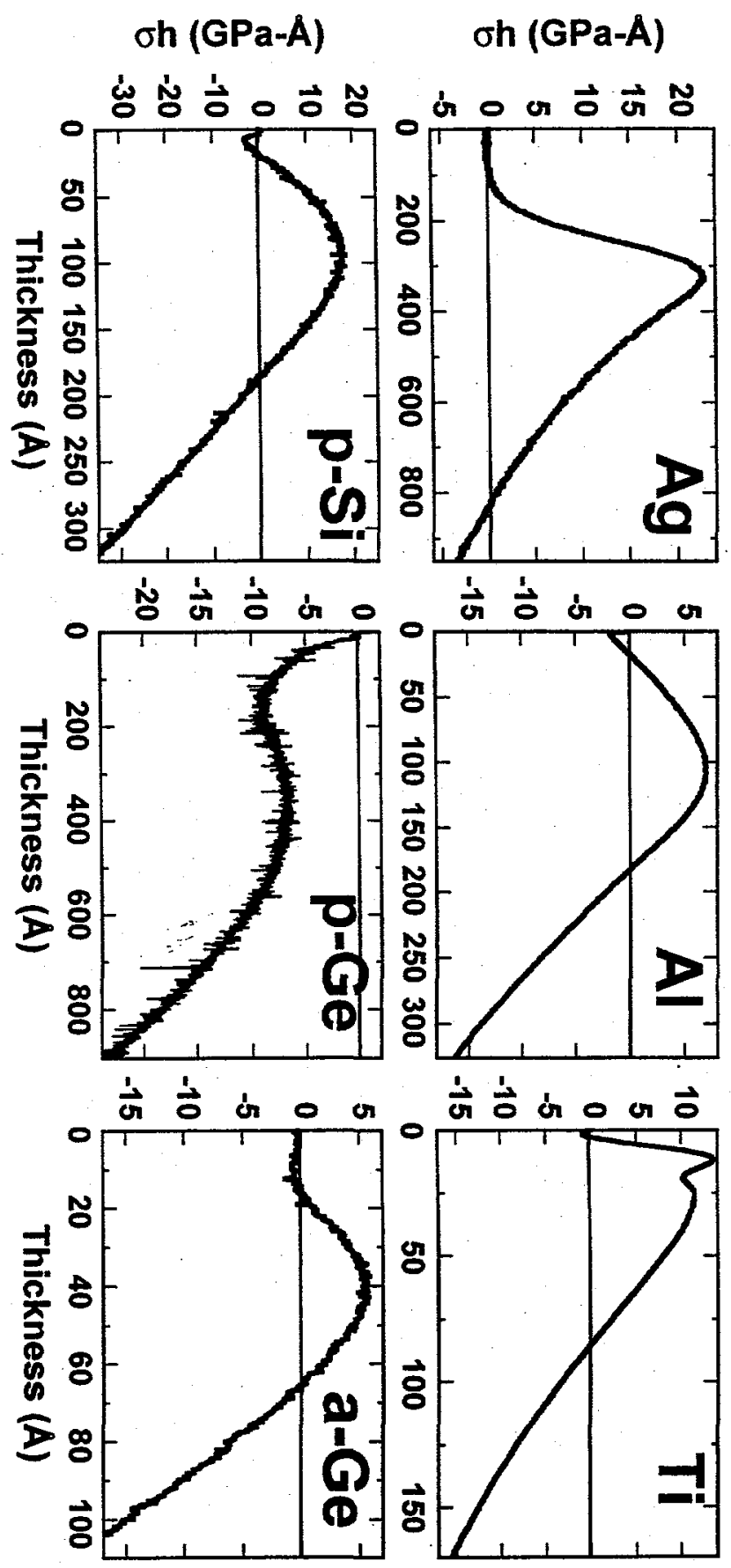



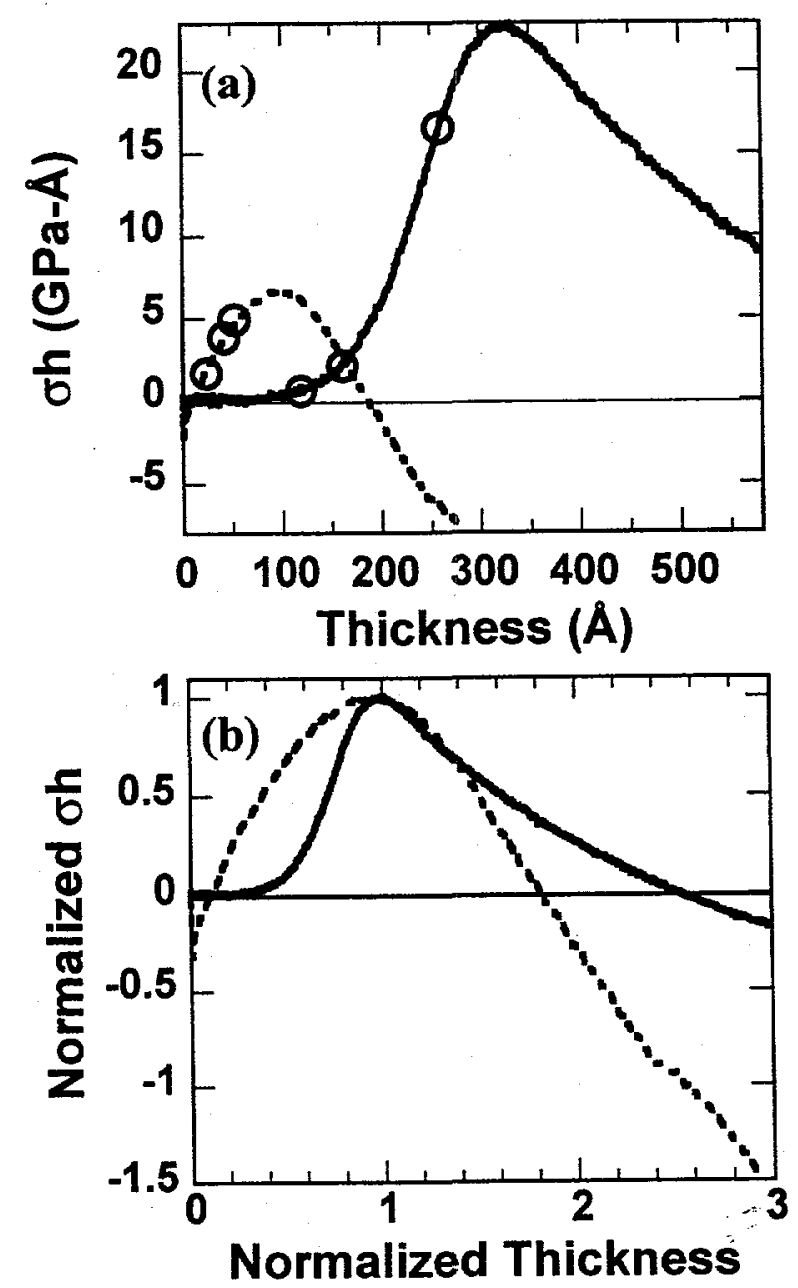

Figure 4

J. A. Floro 


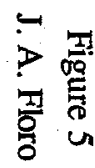

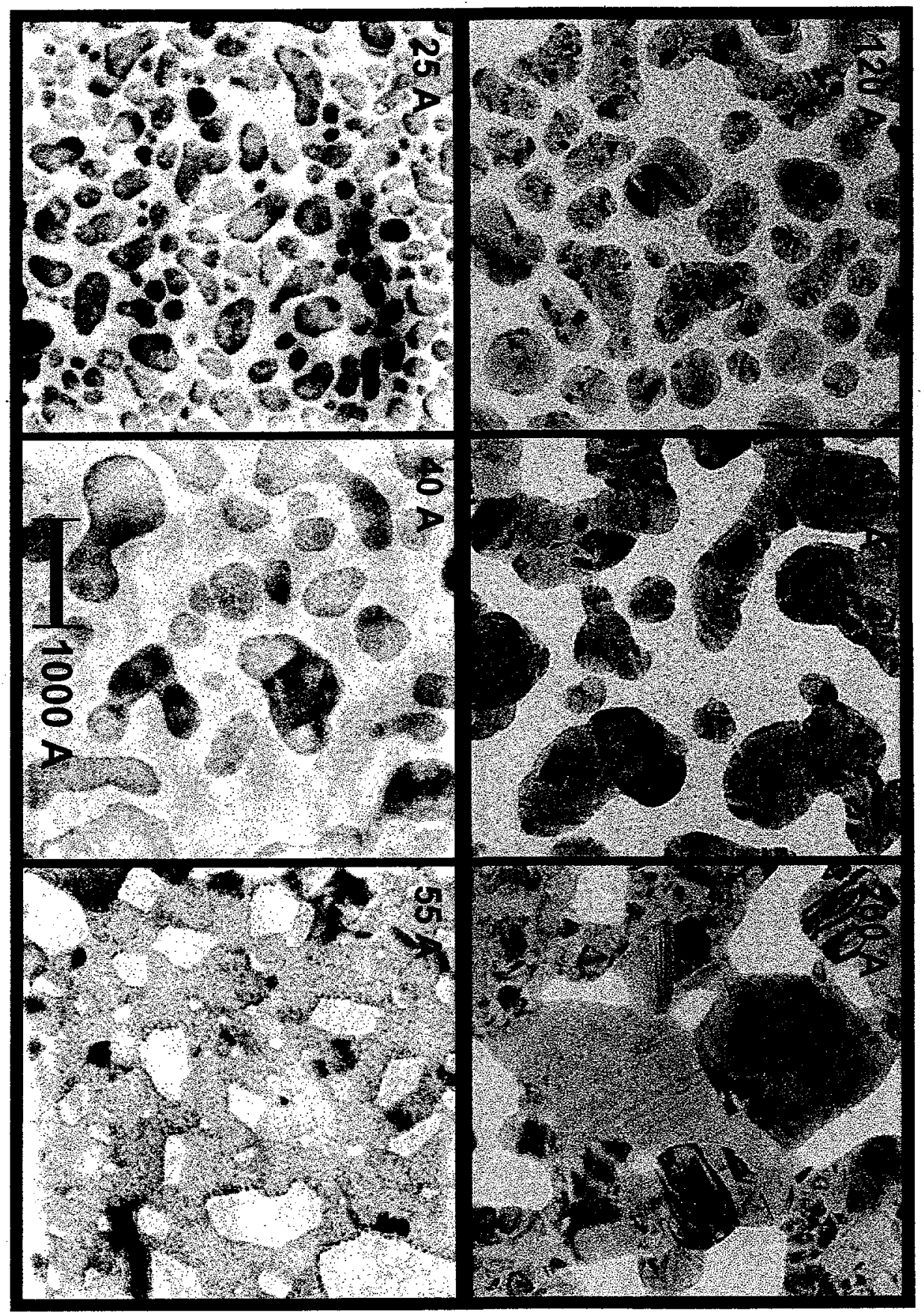




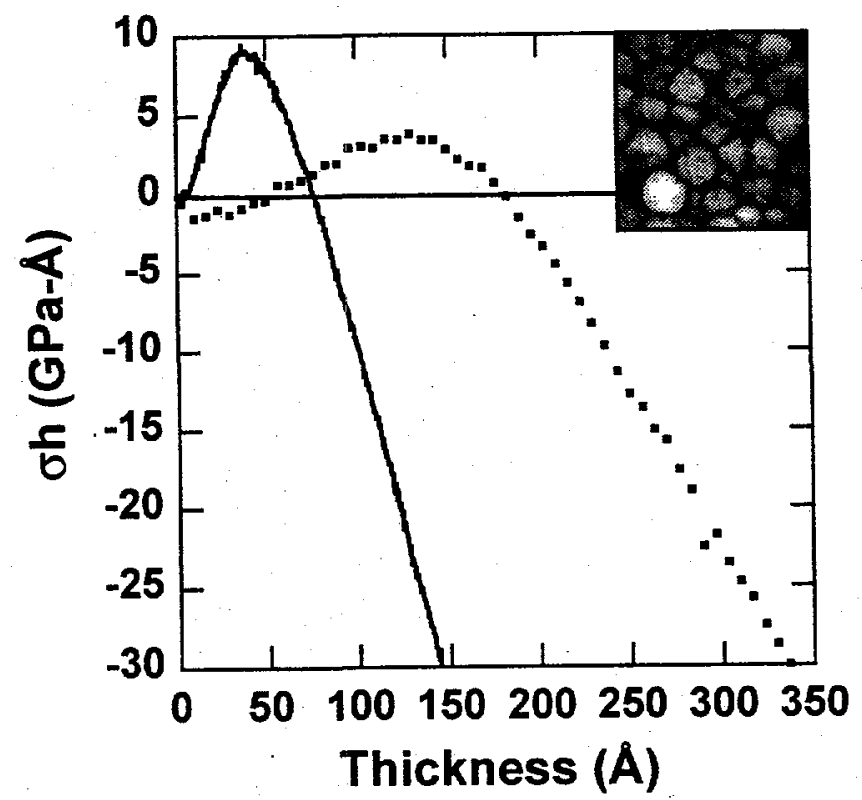

Figure 6

J. A. Floro 

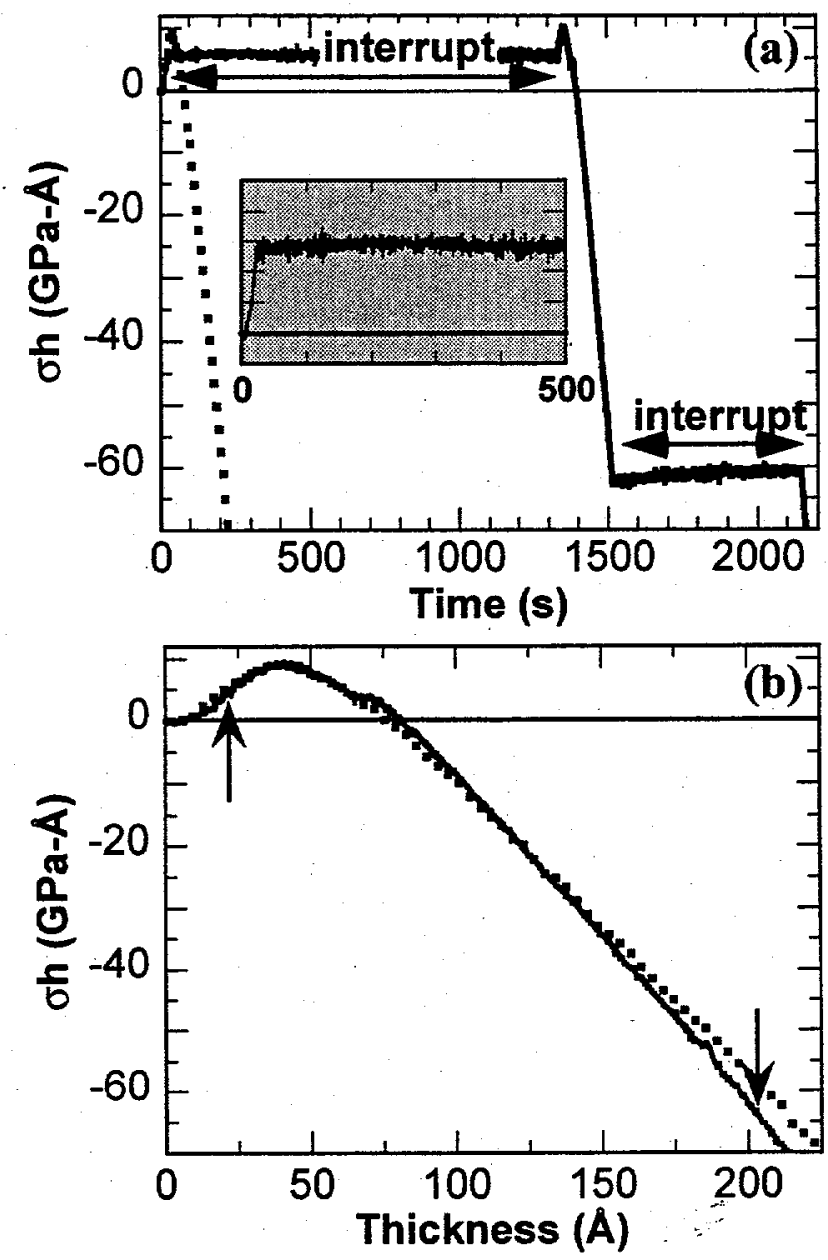

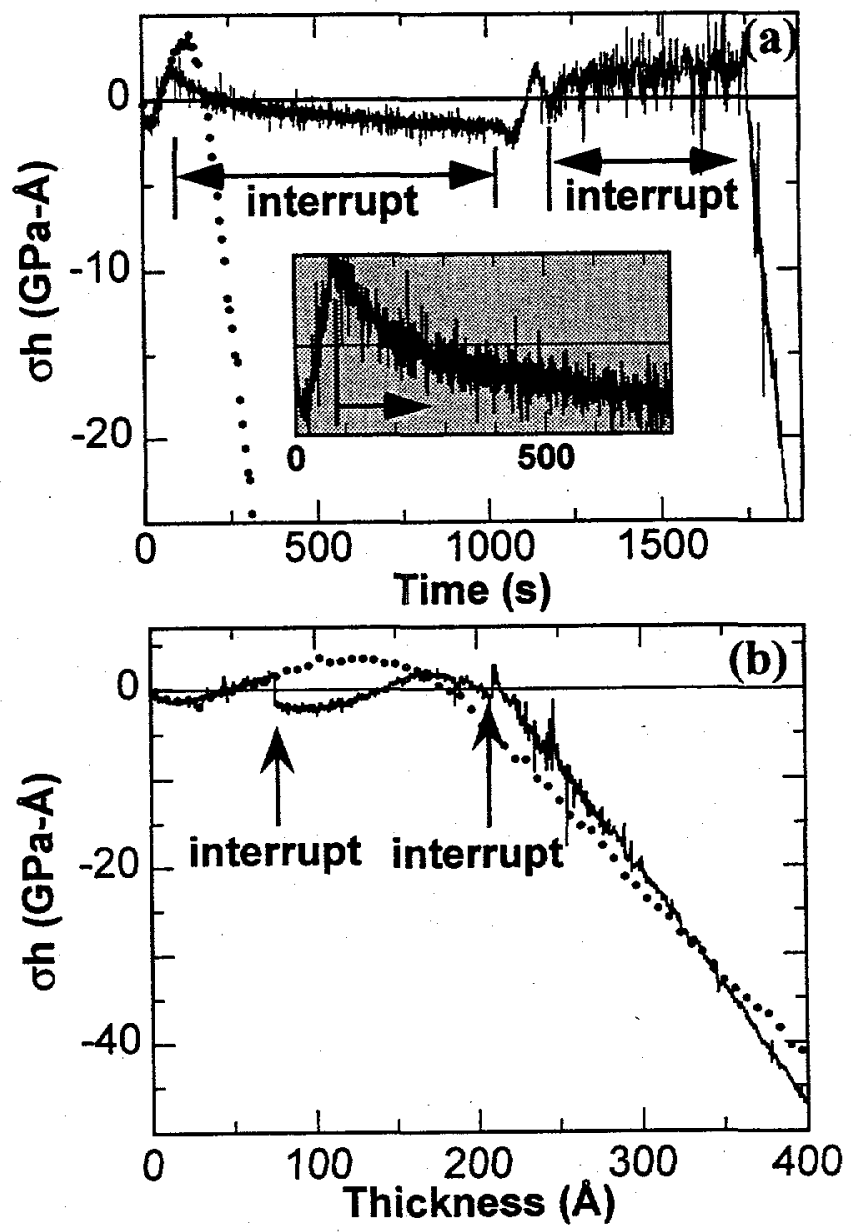

Figure 8

J A. Floro 

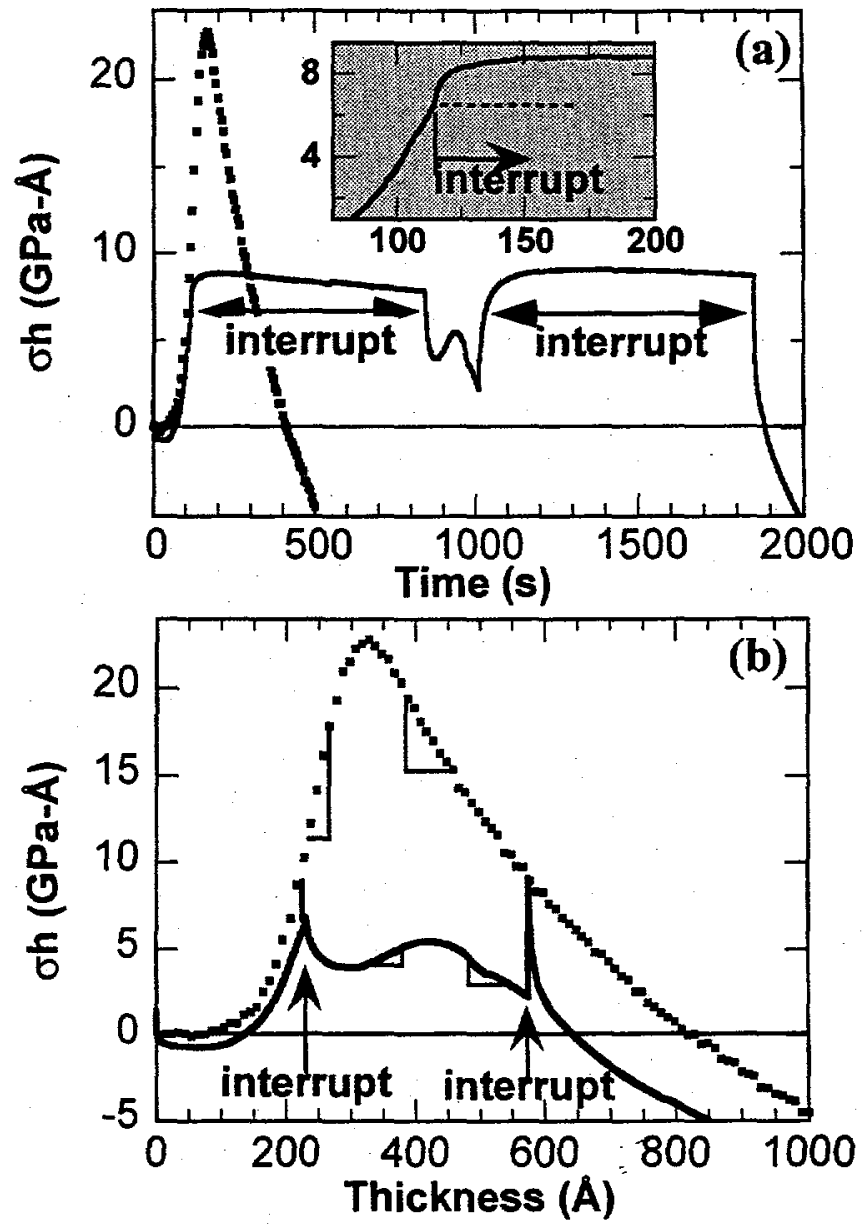

Figure 9

J A. Floro 

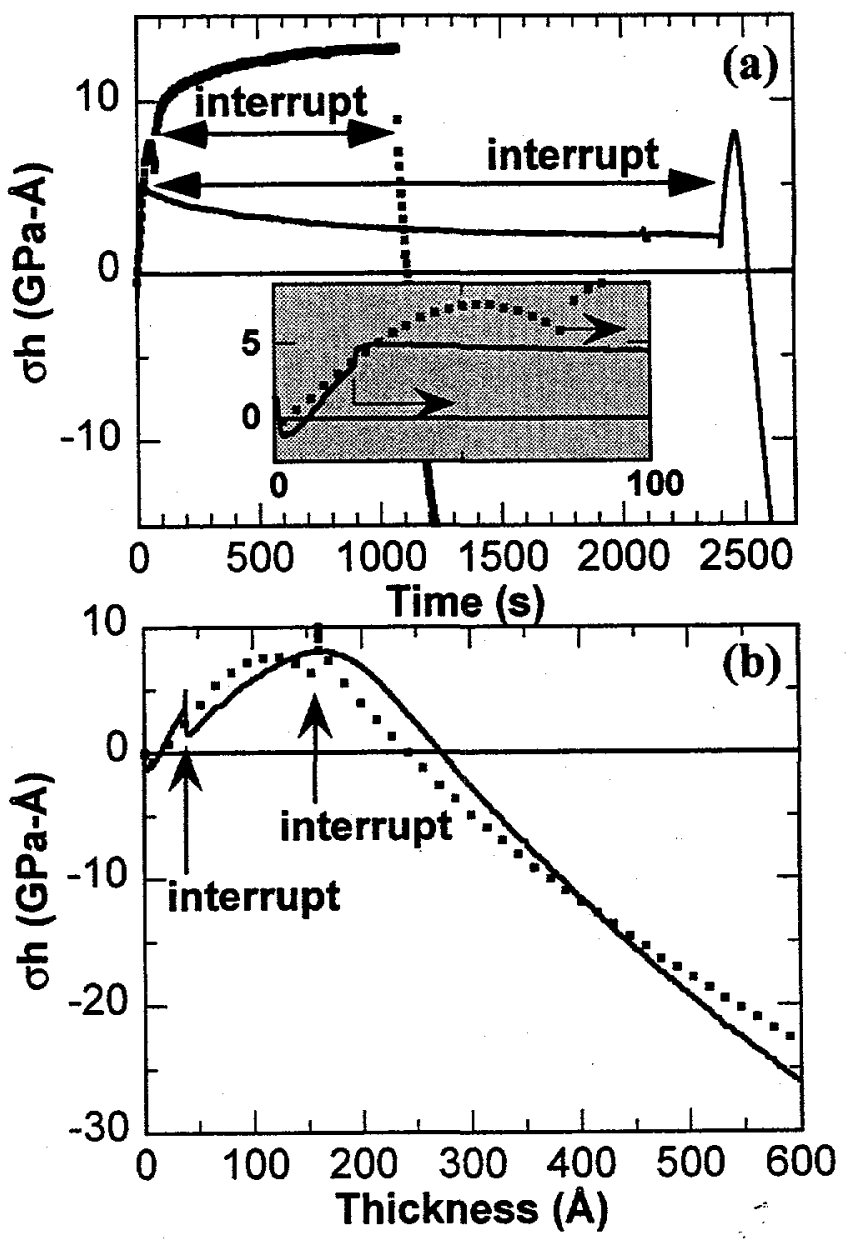


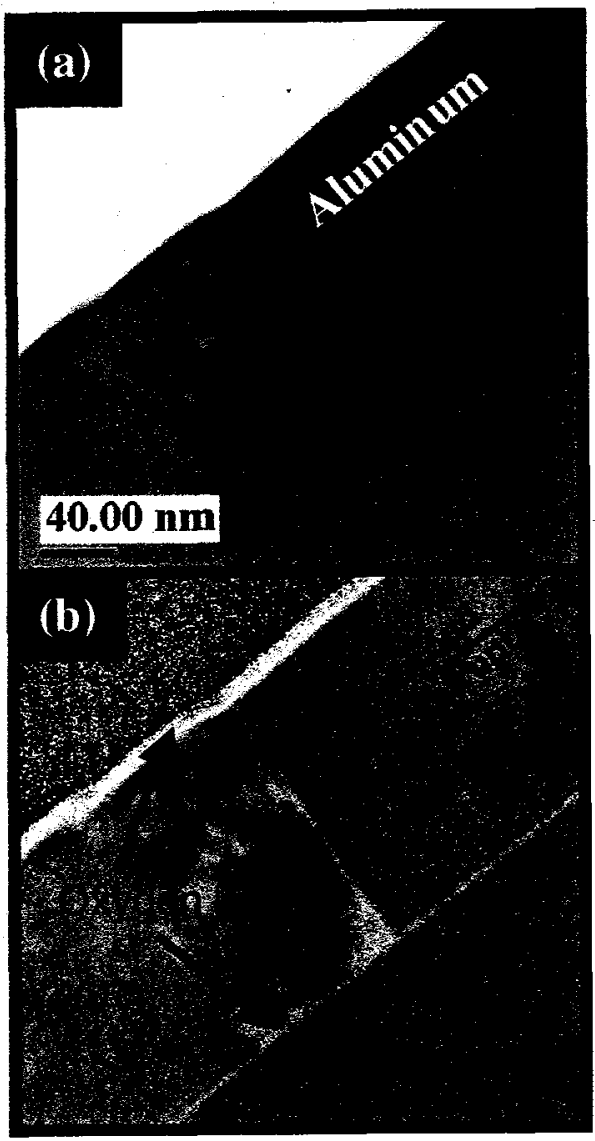

Figure 11

J. A. Floro 

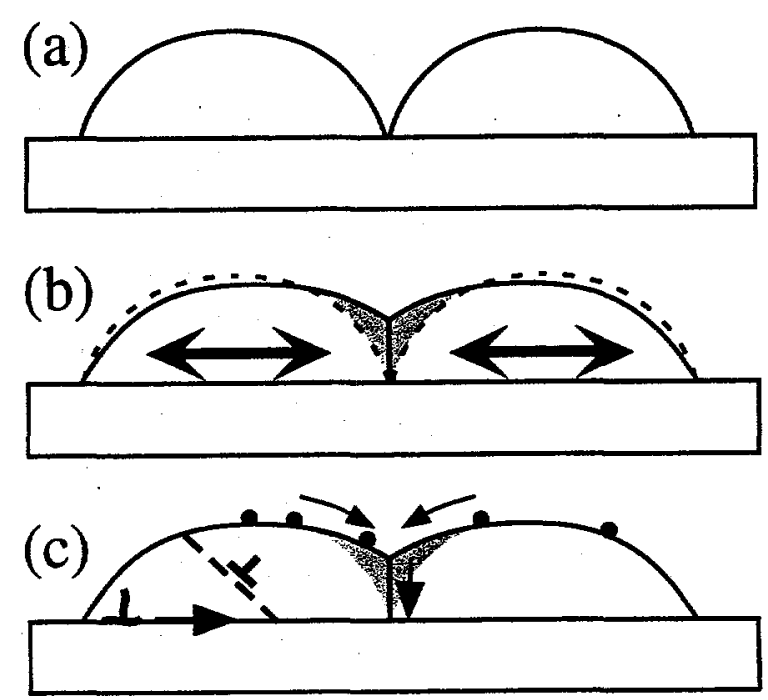

Figure 12

J. A. Floro 


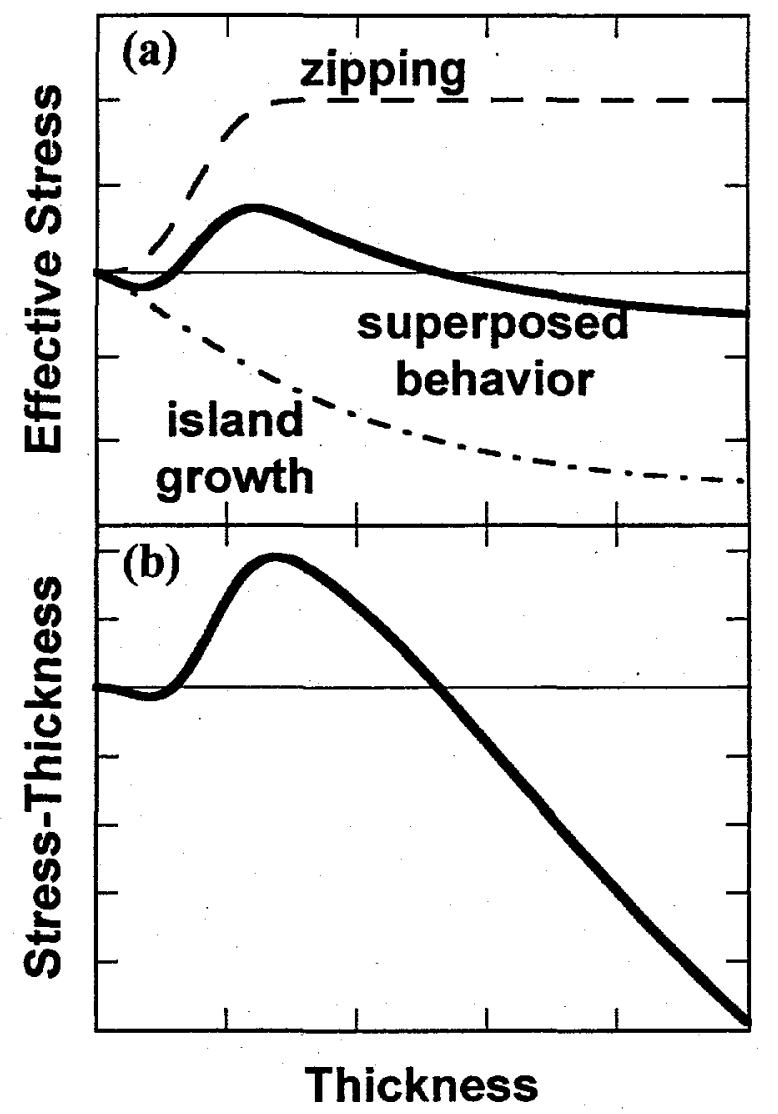

Figure 13

J. A. FLORO 


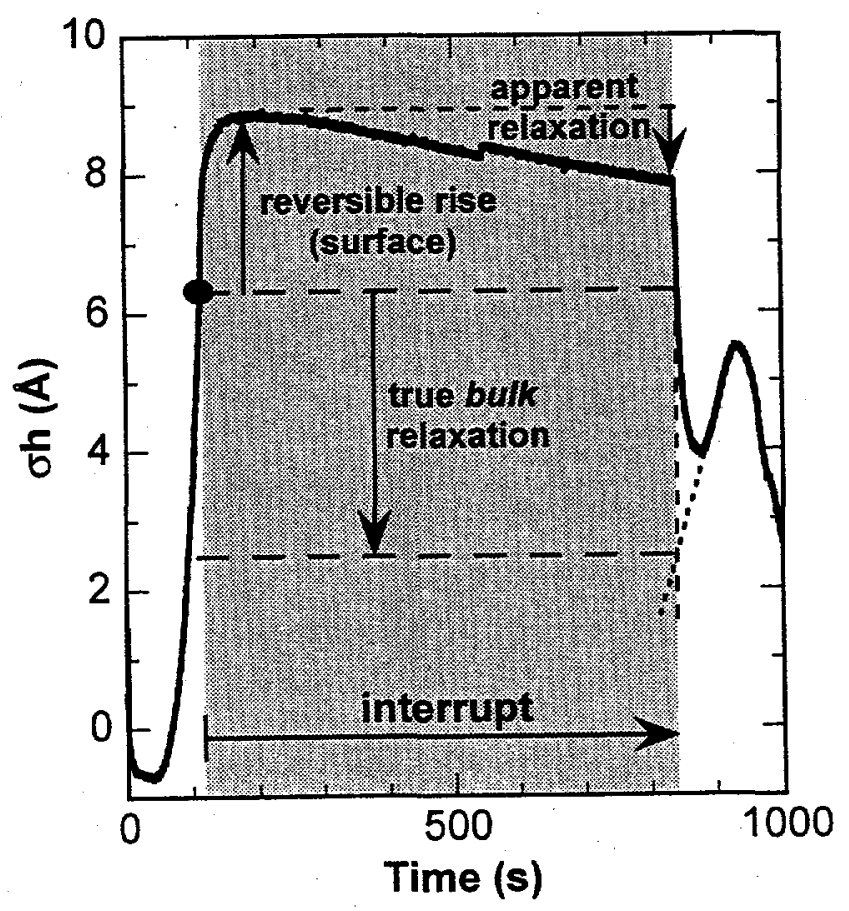

Figure 14

J. A. Floro 